\title{
The Brezis-Nirenberg problem for the Hénon equation: ground state solutions
}

\author{
Simone Secchi* \\ Dipartimento di Matematica ed Applicazioni \\ Università di Milano-Bicocca \\ Via R. Cozzi 53, I-20125 Milano (Italy) \\ e-mail: Simone.Secchi@unimib.it
}

November 13, 2018

\begin{abstract}
This work is devoted to the Dirichlet problem for the equation $-\Delta u=\lambda u+$ $|x|^{\alpha}|u|^{2^{*}-2} u$ in the unit ball of $\mathbb{R}^{N}$. We assume that $\lambda$ is bigger than the first eigenvalues of the laplacian, and we prove that there exists a solution provided $\alpha$ is small enough. This solution has a variational characterization as a ground state.
\end{abstract}

1991 Mathematics Subject Classification. 35J20, 35J61, 35J91.

Key words. Ground states, critical exponent, Hénon equation, Nehari manifold.

\section{Introduction}

This short note is devoted to the Dirichlet problem

$$
\begin{cases}-\Delta u=\lambda u+|x|^{\alpha}|u|^{2^{*}-2} u & \text { in } \Omega \\ u=0 & \text { on } \partial \Omega\end{cases}
$$

where $\Omega$ is the unit ball of $\mathbb{R}^{N}, \lambda$ is bigger than $\lambda_{1}=\lambda_{1}(-\Delta)$, the first Dirichlet eigenvalue of $-\Delta$, and $\alpha$ is a positive parameter. The exponent $2^{*}$ is a shorthand for the Sobolev critical exponent $2 N /(N-2)$. We will assume throughout that $N \geq 3$.

This problem is a generalization of the celebrated Brezis-Nirenberg problem, see [5] and [1, 9, 11, 10] for more general and/or recent existence results. When $\alpha \neq 0$, our equation is reminiscent of the Hénon equation

$$
-\Delta u=|x|^{\alpha}|u|^{p-2} u
$$

*Partially supported by PRIN 2009 "Teoria dei punti critici e metodi perturbativi per equazioni differenziali nonlineari". 
which has been studied deeply in recent times. Most papers deal with the subcritical case $p<(N+2) /(N-2)$, and focus on the behavior of solutions as $\alpha \rightarrow+\infty$ or $p \rightarrow(N+2) /(N-2)$. We refer to [18, 8, 12, 7, 8] for more information. As far as we know, the Brezis-Nirenberg problem for the critical Hénon equation has been studied only in [13], where the authors prove that there always exists a solution to problem (1.1), provided $N \geq 7$ and $\alpha$ is small enough.

In the next sections we will show that solutions exist whenever $N \geq 5$ and $\alpha$ is small; in addition, we will find them as ground-state solutions, in a sense that will be made precise in a moment. We can therefore remove the (technical) restriction on the space dimension, and also provide more information about solutions. We will borrow many ideas from the recent papers [15] and 21], although the presence of the increasing weight $|\cdot|^{\alpha}$ has to be dealt with carefully. Our main result is the following theorem.

Theorem 1.1. Denote by $\lambda_{1}<\lambda_{2} \leq \lambda_{3} \ldots \leq \lambda_{m} \leq \ldots$ the Dirichlet eigenvalues of the laplacian, and assume that $N \geq 5$. If $\lambda_{m} \leq \lambda<\lambda_{m+1}$ for some $m \in \mathbb{N}$, then, for every $\alpha>0$ sufficiently small, there exists (at least) a ground-state solution to problem (1.1).

For the precise definition of ground-state solutions, we refer to Definition 2.1 below.

As a consequence of well-known results in bifurcation theory for potential operators (we refer to Theorem 6.1 of [2]), it is rather easy to prove that each eigenvalue $\lambda_{m}$ is a bifurcation point for problem (1.1): this is the reason why many papers focused on the case $\lambda \notin \sigma(-\Delta)$. We propose a variational approach that also covers the case $\lambda=\lambda_{m} \in \sigma(-\Delta)$.

\section{A variational framework for ground-state solu- tions}

We will work in the Hilbert space $H=H_{0}^{1}(\Omega)$ endowed with the Dirichlet inner product

$$
\langle u, v\rangle=\int_{\Omega} \nabla u \cdot \nabla v
$$

and the induced norm $\|\cdot\|$. We will assume that, for some $m \in \mathbb{N}$,

$$
\lambda_{m} \leq \lambda<\lambda_{m+1},
$$

as stated in Theorem 1.1. We denote by $\left\{e_{j}\right\}_{j}$ the eigenfunctions associated to $\left\{\lambda_{j}\right\}_{j}$. By assumption, we are led to the decomposition

$$
H=Z \oplus Y
$$

where $Z$ is the subspace of $H$ spanned by the first $m$ eigenfunctions $e_{1}, \ldots, e_{m}$ and $Y=Z^{\perp}$. There is a standard identification of solutions to (1.1) with the critical 
points of the functional $\varphi: H \rightarrow \mathbb{R}$ defined by the formula

$$
\varphi(u)=\frac{1}{2} \int_{\Omega}\left(|\nabla u|^{2}-\lambda|u|^{2}\right)-\frac{1}{2^{*}} \int_{\Omega}|x|^{\alpha}|u|^{2^{*}} .
$$

In order to find ground state solutions of (1.1), we introduce (see 15]) a sub-manifold of $H$,

$$
\mathcal{N}=\{u \in H \backslash\{0\} \mid\langle\nabla \varphi(u), u\rangle=0, \nabla \varphi(u) \in Y\}
$$

Remark 2.1. The set $\mathcal{N}$ is the intersection of the standard Nehari manifold

$$
\{u \in H \mid\langle\nabla \varphi(u), u\rangle=0\}
$$

with the pre-image $(\nabla \varphi)^{-1}(Y)$. Much more general cases of Nehari-like manifolds and natural constraints are studied in [14].

Proposition 2.1. The set $\mathcal{N}$ is a $C^{1}$ submanifold of $H$, of codimension $m+1$. Moreover, $\mathcal{N}$ is a natural contraint for $\varphi$ : every critical point of the restriction $\varphi_{\mid \mathcal{N}}$ is a free critical point of $\varphi$.

Proof. We borrow the proof from [21]. Consider the map $F: H \backslash\{0\} \rightarrow \mathbb{R} \times Z$, defined by the formula

$$
F(u)=(\langle\nabla \varphi(u), u\rangle, Q \nabla \varphi(u)),
$$

where $Q$ is the orthogonal projection of $H$ onto $Z$; then $\mathcal{N}=F^{-1}(0)$. On the cartesian product $\mathbb{R} \times Z \simeq \mathbb{R}^{m+1}$ we put the inner product

$$
\left(t_{1}, z_{1}\right) \cdot\left(t_{2}, z_{2}\right)=t_{1} t_{2}+\left\langle z_{1}, z_{2}\right\rangle .
$$

We claim that

$$
(D F(u)(t u+z)) \cdot(t, z)<0
$$

for any $(t, z) \in \mathbb{R} \times Z,(t, z) \neq(0,0)$. It is elementary to realize that this claim completes the proof of the first part of our Proposition. Fix $(t, z) \neq(0,0)$, and remark that

$$
\langle\nabla \varphi(u), u\rangle=\langle\nabla \varphi(u), z\rangle=0
$$

implies

$$
\begin{gathered}
(D F(u)(t u+z)) \cdot(t, z) \\
=t D^{2} \varphi(u)(t u+z, u)+t\langle\nabla \varphi(u), t u+z\rangle+D^{2} \varphi(u)(t u+z, z) \\
=D^{2} \varphi(u)(t u+z, t u+z)-t\langle\nabla \varphi(u), t u+2 z\rangle \\
=\int_{\Omega}|\nabla z|^{2}-\lambda|z|^{2} d x-\int_{\Omega}\left(\left(2^{*}-1\right)(t u+z)^{2}-t u(t u+2 z)\right)|u|^{2^{*}-2}|x|^{\alpha} d x \\
=\int_{\Omega}|\nabla z|^{2}-\lambda|z|^{2} d x \\
-\int_{\Omega}\left(\left(2^{*}-2\right) t^{2} u^{2}+2\left(2^{*}-2\right) t z u+\left(2^{*}-1\right) z^{2}\right)|u|^{2^{*}-2}|x|^{\alpha} d x .
\end{gathered}
$$


As a quadratic form in $(t, z)$, the integral

$$
\int_{\Omega}\left(\left(2^{*}-2\right) t^{2} u^{2}+2\left(2^{*}-2\right) t z u+\left(2^{*}-1\right) z^{2}\right)|u|^{2^{*}-2}|x|^{\alpha} d x
$$

is positive definite whenever $u(x) \neq 0$. By the assumption $\lambda_{m} \leq \lambda<\lambda_{m+1}$, the quadratic form $\int_{\Omega}|\nabla z|^{2}-\lambda|z|^{2}$ is negative semidefinite. If $\int_{\Omega}|\nabla z|^{2}-\lambda|z|^{2}<0$, the claim is proved. If $\int_{\Omega}|\nabla z|^{2}-\lambda|z|^{2}=0$, either $\lambda=\lambda_{m}$ and $z$ is an eigenfunction, or $z=0$. By assumption, $t \neq 0$ if $z=0$; moreover, $z \neq 0$ implies $z \neq 0$ almost everywhere. In both cases, the claim follows easily.

Finally, we need to check that $u \in H$ is a critical point of $\varphi$ if and only if $u \in \mathcal{N}$ and $D \varphi(u)$ vanishes on the tangent space $T_{u} \mathcal{N}$. The necessary condition is trivial; on the contrary, assuming that $D \varphi(u)=0$ on $T_{u} \mathcal{N}$ and $u \in \mathcal{N}$, we deduce that $D \varphi(u)$ also vanishes on $\mathbb{R} u \oplus Z$. But we have just proved that $\mathbb{R} u \oplus Z$ is transversal to $T_{u} \mathcal{N}$, and we conclude.

Remark 2.2. The previous Proposition states that $D F(u)$ is a surjective map at every $u \in F^{-1}(0) \backslash\{0\}$. But the additional information that $(D F(u)(t u+z)) \cdot(t, z)$ is negative will be useful later on.

Since $\mathcal{N}$ contains every critical point of $\varphi$, the following terminology is rather natural.

Definition 2.1. A ground state solution $u$ to (1.1) is any element of $\mathcal{N}$ such that $D \varphi(u)$ vanishes on $T_{u} \mathcal{N}$ and $\varphi(u)=c$, where the level $c$ is defined by

$$
c=\inf _{\mathcal{N}} \varphi
$$

The arguments of [20], which hold true under general assumptions, guarantee that for every $v \in Y \backslash\{0\}$ there exists a unique couple $(f(v), g(v)) \in(0,+\infty) \times Z$ such that $F(f(v) v+g(v))=0$. Moreover $f(\cdot)$ and $g(\cdot)$ are continuous maps, and

$$
\varphi(f(v) v+g(v))=\max _{\substack{t>0 \\ w \in Z}} \varphi(t v+w) .
$$

It follows easily from the definition of $f$ and $g$ that

$$
c=\inf _{\substack{v \in Y \\ v \neq 0}} \varphi(f(v) v+g(v))=\inf _{\substack{v \in Y \\ v \neq 0}} \max _{\substack{t>0 \\ w \in Z}} \varphi(t v+w) .
$$

\section{Existence of ground state solutions}

The existence of a ground state solution to (1.1) will be proved by a compactness argument. Since (1.1) contains the critical exponent, it is natural to expect compactness of minimizing sequences (for $c$ ) below some energy level related to Sobolev's best constant $S$. Recall that

$$
S=\inf _{\substack{u \in H \\ u \neq 0}} \frac{\int_{\Omega}|\nabla u|^{2} d x}{\left(\int_{\Omega}|u|^{2^{*}} d x\right)^{2 / 2^{*}}},
$$


and this numer is actually indipendent of the domain $\Omega$. A simple exercise in sophomore calculus proves the next lemma, stated in [21].

Lemma 3.1. If $A>0$ and $B>0$, then

$$
\max _{t>0}\left(\frac{1}{2} A t^{2}-\frac{1}{2^{*}} B t^{2^{*}}\right)=\frac{1}{N}\left(\frac{A}{B^{2 / 2^{*}}}\right)^{N / 2} .
$$

We now come to the main compactness result about the variational problem (2.3).

Proposition 3.1. Suppose that

$$
c<\frac{1}{N} S^{N / 2} .
$$

Then there exists $v \in Y \backslash\{0\}$ such that

$$
\max _{\substack{t>0 \\ w \in Z}} \varphi(t v+w)=\varphi(f(v) v+g(v))=c .
$$

Proof. Take any sequence $\left\{v_{n}\right\}_{n}$ in $Y \backslash\{0\}$ such that $\left\|v_{n}\right\|=1$ and

$$
\max _{\substack{t>0 \\ w \in Z}} \varphi\left(t v_{n}+w\right) \rightarrow c .
$$

Without loss of generality, we can assume that $v_{n} \rightarrow v$ weakly in $H$, strongly in $L^{2}(\Omega)$ and point-wise almost everywhere. Writing

$$
\begin{aligned}
A & =\lim _{n \rightarrow+\infty} \int_{\Omega}\left|\nabla\left(v_{n}-v\right)\right|^{2} d x \\
B_{\alpha} & =\lim _{n \rightarrow+\infty} \int_{\Omega}\left|v_{n}-v\right|^{2^{*}}|x|^{\alpha} d x
\end{aligned}
$$

and using the Brezis-Nirenberg lemma, we exploit (3.2) to get

$$
\varphi(t v+w)+\frac{1}{2} A t^{2}-\frac{1}{2^{*}} B_{\alpha} t^{2^{*}} \leq c .
$$

We now distinguish several possibilities. If $v=0$ and $B_{\alpha}=0$, from the assumption $\left\|v_{n}\right\|=1$ we deduce $A=1$. Hence $t^{2} \leq 2 c$ for every $t>0$, a contradiction.

Assume now $B_{\alpha} \neq 0$. From the Sobolev inequality and the trivial remark that $|x|^{\alpha}<1$ in $\Omega$, we get

$$
\frac{1}{N} S^{N / 2} \leq \frac{1}{N}\left(\frac{A}{B_{0}^{2 / 2^{*}}}\right)^{\frac{N}{2}} \leq \frac{1}{N}\left(\frac{A}{B_{\alpha}^{2 / 2^{*}}}\right)^{\frac{N}{2}}=\max _{t>0}\left(\frac{1}{2} A t^{2}-\frac{1}{2^{*}} B_{\alpha} t^{2^{*}}\right) .
$$

If $v=0$, we conclude that

$$
\frac{1}{N} S^{N / 2} \leq c<\frac{1}{N} S^{N / 2},
$$


and thus $v \neq 0$. Call $h=g(v) / f(v)$. It follows from the definition of the level $c$ that

$$
\begin{aligned}
c \leq \varphi(f(v)(v+h))=\max _{t>0} \varphi(t(v+h)) & \\
& =\frac{1}{N} \frac{\int_{\Omega}|\nabla v|^{2}+|\nabla h|^{2}-\lambda\left(v^{2}+h^{2}\right) d x}{\left(\int_{\Omega}|v+h|^{2^{*}}|x|^{\alpha} d x\right)^{2 / 2^{*}}} .
\end{aligned}
$$

From (3.3),

$$
\begin{aligned}
\max _{t>0}\left(\varphi(t(v+h))+\frac{1}{2} A t^{2}\right. & \left.-\frac{1}{2^{*}} B_{\alpha} t^{2^{*}}\right) \\
& =\frac{1}{N} \frac{A+\int_{\Omega}|\nabla v|^{2}+|\nabla h|^{2}-\lambda\left(v^{2}+h^{2}\right) d x}{\left(B_{\alpha}+\int_{\Omega}|v+h|^{2^{*}}|x|^{\alpha} d x\right)^{2 / 2^{*}}} \leq c .
\end{aligned}
$$

Putting together (3.1), (3.4), (3.5) and (3.6) we can write

$$
\begin{gathered}
(N c)^{2 / N}\left(B_{\alpha}+\int_{\Omega}|v+h|^{2^{*}}|x|^{\alpha} d x\right)^{2 / 2^{*}} \\
<(N c)^{2 / N}\left(B_{\alpha}^{2 / 2^{*}}+\left(\int_{\Omega}|v+h|^{2^{*}}|x|^{\alpha} d x\right)^{2 / 2^{*}}\right) \\
<A+\int_{\Omega}|\nabla v|^{2}+|\nabla h|^{2}-\lambda\left(v^{2}+h^{2}\right) d x \\
\leq(N c)^{2 / N}\left(B_{\alpha}+\int_{\Omega}|v+h|^{2^{*}}|x|^{\alpha} d x\right)^{2 / 2^{*}}
\end{gathered}
$$

a contradiction. Therefore $B_{\alpha}=0$ and (3.3) yields

$$
c \leq \varphi(f(v) v+g(v)) \leq c .
$$

Remark 3.1. It was proved in [15], mimicking the ideas contained in [5], that $\varphi$ satisfies the Palais-Smale condition below the threshold $S^{N / 2} / N$. The same result could also be proved by slightly adapting the arguments of [16].

The subspace $Z$ has a kind of unique continuation property, as proved in [21, Lemma 3.3].

Lemma 3.2. If $w \in Z$ vanishes on some open subset $\omega \neq \emptyset$ of $\Omega$, then $w=0$ everywhere.

The next step is to check that the level $c$ defined in (2.3) satisfies inequality (3.1). We proceed in several steps.

Consider $\ell \ll 1$, a parameter that will tend to zero at a slower rate than $\varepsilon$ : $\varepsilon / \ell \rightarrow 0$. As $\ell \rightarrow 0$, the point

$$
x_{\ell}=(1-\ell, 0, \ldots, 0)
$$


approaches the boundary of $\Omega$. We pick a test function $\xi=\xi_{\ell} \in C_{0}^{\infty}(\Omega)$ spiked at $x_{\ell}$ :

$$
\xi(x)= \begin{cases}1, & \text { if } x \in B\left(x_{\ell}, \ell / 2\right) \\ 0, & \text { if } x \notin B\left(x_{\ell}, \ell\right),\end{cases}
$$

and such that $\left|\nabla \xi_{\ell}\right| \leq C / \ell$. It is well known that the instanton $U_{\varepsilon}$ defined by the formula

$$
U_{\varepsilon, \ell}(x)=(N(N-2))^{\frac{N-2}{4}} \frac{\varepsilon^{\frac{N-2}{2}}}{\left(\varepsilon^{2}+\left|x-x_{\ell}\right|^{2}\right)^{\frac{N-2}{2}}}
$$

is the optimal function for the Sobolev inequality in

$$
D^{1,2}\left(\mathbb{R}^{N}\right)=\left\{u \in L^{2^{*}}\left(\mathbb{R}^{N}\right) \mid \nabla u \in L^{2}\left(\mathbb{R}^{N}\right)\right\} .
$$

Call now

$$
u_{\varepsilon, \ell}(x)=\xi_{\ell}(x) U_{\varepsilon, \ell}(x) .
$$

Up to a constant that we can neglect in the following estimates, we can pretend that

$$
u_{\varepsilon, \ell}(x)=\xi_{\ell}(x) \frac{\varepsilon^{\frac{N-2}{2}}}{\left(\varepsilon^{2}+\left|x-x_{\ell}\right|^{2}\right)^{\frac{N-2}{2}}} .
$$

Reasoning as in [5], we can estimates

$$
\int_{\Omega}\left|\nabla u_{\varepsilon, \ell}\right|^{2} d x=S^{\frac{N}{2}}+\text { h.o.t. }
$$

where h.o.t denotes higher order terms like

$$
\begin{aligned}
\varepsilon^{N-2} \int_{B\left(x_{\ell}, \ell\right) \backslash B\left(x_{\ell}, \ell / 2\right)} \frac{\left|\nabla \xi_{\ell}(x)\right|^{2}}{\left(\varepsilon^{2}+\left|x-x_{\ell}\right|^{2}\right)^{N-2}} d x & \\
\leq C & \frac{\varepsilon^{N-2}}{\ell^{2}} \int_{B\left(x_{\ell}, \ell\right) \backslash B\left(x_{\ell}, \ell / 2\right)} \frac{d x}{\left(\varepsilon^{2}+\left|x-x_{\ell}\right|^{2}\right)^{N-2}} \\
= & C \frac{\varepsilon^{N-2}}{\ell^{2}} \int_{B(0, \ell) \backslash B(0, \ell / 2)} \frac{d y}{\left(\varepsilon^{2}+|y|^{2}\right)^{N-2}} \\
\leq C \frac{\varepsilon^{N-2}}{\ell^{2}} \int_{B(0, \ell) \backslash B(0, \ell / 2)} \frac{d y}{\left(\varepsilon^{2}+\frac{\ell^{2}}{16}\right)^{N-2}} & =C \frac{\varepsilon^{N-2}}{\ell^{2}} \frac{\ell^{N}}{\ell^{2(N-2)}}=C\left(\frac{\varepsilon}{\ell}\right)^{N-2}
\end{aligned}
$$

As a consequence,

$$
\int_{\Omega}\left|\nabla u_{\varepsilon, \ell}\right|^{2} d x=S^{\frac{N}{2}}+O\left(\left(\frac{\varepsilon}{\ell}\right)^{N-2}\right) .
$$


Similarly,

$$
\begin{aligned}
\int_{\Omega}\left|u_{\varepsilon, \ell}\right|^{2^{*}}= & \varepsilon^{N} \int_{\Omega} \frac{\left|\xi_{\ell}(x)\right|^{2^{*}}}{\left(\varepsilon^{2}+\left|x-x_{\ell}\right|^{2}\right)^{N}} \\
= & \varepsilon^{N} \int_{\Omega} \frac{\left|\xi_{\ell}(x)\right|^{2^{*}}-1}{\left(\varepsilon^{2}+\left|x-x_{\ell}\right|^{2}\right)^{N}} d x+\varepsilon^{N} \int_{\Omega} \frac{d x}{\left(\varepsilon^{2}+\left|x-x_{\ell}\right|^{2}\right)^{N}} \\
& =\int_{\mathbb{R}^{N}}\left|U_{\varepsilon, \ell}\right|^{2^{*}}+O\left(\varepsilon^{N}\right)+\varepsilon^{N} \int_{\Omega} \frac{\left|\xi_{\ell}(x)\right|^{2^{*}}-1}{\left(\varepsilon^{2}+\left|x-x_{\ell}\right|^{2}\right)^{N}} d x .
\end{aligned}
$$

But

$$
\begin{array}{r}
\varepsilon^{N} \int_{\Omega} \frac{\left|\xi_{\ell}(x)\right|^{2^{*}}-1}{\left(\varepsilon^{2}+\left|x-x_{\ell}\right|^{2}\right)^{N}} d x=\varepsilon^{N} \int_{\Omega \backslash B\left(x_{\ell}, \ell / 2\right)} \frac{\left|\xi_{\ell}(x)\right|^{2^{*}}-1}{\left(\varepsilon^{2}+\left|x-x_{\ell}\right|^{2}\right)^{N}} d x \\
\leq C \frac{\varepsilon^{N}}{\ell^{2 N}} \ell^{N}=C\left(\frac{\varepsilon}{\ell}\right)^{N} .
\end{array}
$$

We conclude that

$$
\int_{\Omega}\left|u_{\varepsilon, \ell}\right|^{2^{*}}=\int_{\mathbb{R}^{N}}\left|U_{\varepsilon, \ell}\right|^{2^{*}}+O\left(\varepsilon^{N}\right)+O\left(\left(\frac{\varepsilon}{\ell}\right)^{N}\right) .
$$

The $L^{2}$-norm is slightly more involved:

$$
\begin{aligned}
\int_{\Omega}\left|u_{\varepsilon, \ell}\right|^{2}=\varepsilon^{N-2} \int_{B\left(x_{\ell}, \ell\right)} \frac{d x}{\left(\varepsilon^{2}+\mid\right.} & \left.x-\left.x_{\ell}\right|^{2}\right)^{N-2} \\
& +\varepsilon^{N-2} \int_{B\left(x_{\ell}, \ell\right) \backslash B\left(x_{\ell}, \ell / 2\right)} \frac{\xi_{\ell}(x)^{2}}{\left(\varepsilon^{2}+\left|x-x_{\ell}\right|^{2}\right)^{N-2}} .
\end{aligned}
$$

Now,

$$
\begin{aligned}
\varepsilon^{N-2} \int_{B\left(x_{\ell}, \ell\right) \backslash B\left(x_{\ell}, \ell / 2\right)} \frac{\xi_{\ell}(x)^{2}}{\left(\varepsilon^{2}+\left|x-x_{\ell}\right|^{2}\right)^{N-2}} \leq C \varepsilon^{N-2} \int_{\ell / 2}^{\ell} r^{3-N} d r & \\
& =C \frac{\varepsilon^{N}-2}{\ell^{N-4}} .
\end{aligned}
$$

On the other hand,

$$
\begin{gathered}
\varepsilon^{N-2} \int_{B\left(x_{\ell}, \ell\right)} \frac{d x}{\left(\varepsilon^{2}+\left|x-x_{\ell}\right|^{2}\right)^{N-2} \geq C \varepsilon^{N-2}} \int_{B\left(x_{\ell}, \varepsilon\right)} \frac{d x}{\left(2 \varepsilon^{2}\right)^{N-2}}+ \\
C \varepsilon^{N-2} \int_{B\left(x_{\ell}, \ell\right) \backslash B\left(x_{\ell}, \varepsilon\right)} \frac{d x}{\left(2\left|x-x_{\ell}\right|^{2}\right)^{N-2}}=C \varepsilon^{2}+C \varepsilon^{N-2} \int_{\varepsilon}^{\ell} r^{3-N} d r \\
=C \varepsilon^{2}+O\left(\frac{\varepsilon^{N-2}}{\ell^{N-4}}\right) .
\end{gathered}
$$


We are now ready to estimate

$$
\begin{array}{r}
\frac{\int_{\Omega}\left|\nabla u_{\varepsilon, \ell}\right|^{2}-\lambda\left|u_{\varepsilon, \ell}\right|^{2}}{\left(\int_{\Omega}|x|^{\alpha}\left|u_{\varepsilon, \ell}\right|^{2^{*}}\right)^{2 / 2^{*}}} \leq \frac{S^{\frac{N}{2}}+O\left(\left(\frac{\varepsilon}{\ell}\right)^{N-2}\right)-\lambda\left(C \varepsilon^{2}+O\left(\frac{\varepsilon^{N-2}}{\ell^{N-4}}\right)\right)}{(1-2 \ell)^{\alpha \frac{2}{2^{*}}}\left(S^{\frac{N}{2}}+O\left((\varepsilon / \ell)^{N}\right)\right)^{2 / 2^{*}}} \\
=\frac{1}{(1-2 \ell)^{\frac{2 \alpha}{2 *}}}\left(S-C \varepsilon^{2}+O\left(\left(\frac{\varepsilon}{\ell}\right)^{N-2}\right)\right)
\end{array}
$$

Proposition 3.2. There results

$$
c<\frac{1}{N} S^{\frac{N}{2}} .
$$

Proof. We will check that

$$
\max _{\substack{t>0 \\ w \in Z}} \varphi\left(t u_{\varepsilon, \ell}+w\right)<\frac{1}{N} S^{N / 2} .
$$

Setting $\omega=\Omega \backslash \operatorname{supp} \xi_{\ell}$, Lemma 3.2 implies that $w \mapsto\|w\|_{L^{2^{*}(\omega)}}$ defines a norm on the subspace $Z$. Since $\operatorname{dim} Z=m<+\infty$, all norms on $Z$ are equivalent: we will use this remark tacitly in the sequel.

We choose $\ell=\sqrt[4]{\varepsilon}$, and write $u_{\varepsilon}$ instead of $u_{\varepsilon, \ell}$. By elementary convexity and recalling that $0 \notin \omega$ so that $|x|^{\alpha}$ is bounded away from zero as $x \in \omega$, for every $t>0$ and every $w \in Z$, we can estimate

$$
\begin{aligned}
\int_{\Omega}\left|t u_{\varepsilon}+w\right|^{2^{*}}|x|^{\alpha} d x=\int_{\Omega \backslash \omega}\left|t u_{\varepsilon}+w\right|^{2^{*}}|x|^{\alpha} d x+\int_{\omega}|w|^{2^{*}}|x|^{\alpha} d x \\
\geq t^{2^{*}} \int_{\Omega}\left|u_{\varepsilon}\right|^{2^{*}}|x|^{\alpha} d x+2^{*} t^{2^{*}-1} \int_{\Omega}\left|u_{\varepsilon}\right|^{2^{*}-1} w|x|^{\alpha} d x+2^{*} C_{1}\|w\|^{2^{*}} .
\end{aligned}
$$

It follows that

$$
\begin{aligned}
\varphi\left(t u_{\varepsilon}+\right. & w) \leq \varphi\left(t u_{\varepsilon}\right)+t \int_{\Omega} \nabla u_{\varepsilon} \cdot \nabla w-\lambda u_{\varepsilon, \ell} w d x \\
& +\frac{1}{2} \int_{\Omega}|\nabla w|^{2}-\lambda|w|^{2} d x-t^{2^{*}-1} \int_{\Omega}\left|u_{\varepsilon}\right|^{2^{*}-1} w|x|^{\alpha} d x-C_{1}\|w\|^{2^{*}} .
\end{aligned}
$$

In particular, we can write

$$
\varphi\left(t u_{\varepsilon}+w\right) \leq A\left(t^{2}+t\|w\|+t^{2^{*}-1}\|w\|\right)-B\left(t^{2^{*}}+\|w\|^{2^{*}}\right)
$$

for suitable constants $A>0$ and $B>0$. Hence there exists a number $R>0$ such that, for $\varepsilon$ and $\ell$ small, $t>R$ and $w \in Z$ there holds $\varphi\left(t u_{\varepsilon}+w\right) \leq 0$. On the other hand, whenever $t \leq R$,

$$
\varphi\left(t u_{\varepsilon}+w\right) \leq \varphi\left(t u_{\varepsilon}\right)+O\left(\varepsilon^{\frac{N-2}{2}}\right)\|w\|-C_{1}\|w\|^{2^{*}} \leq \varphi\left(t u_{\varepsilon}\right)+O\left(\varepsilon^{N \frac{N-2}{2}}\right) .
$$


The last estimate follows from the Young inequality

$$
\max _{s>0}\left(r s-\frac{s^{p}}{2}\right)=\frac{p-1}{p} r^{\frac{p}{p-1}}, \quad p>1 .
$$

We remark that $N(N-2) /(N+2)>2$ since $N \geq 5$. It now follows from Lemma 3.1 and (3.11) that, for $\alpha$ and $\varepsilon$ sufficiently small,

$$
\begin{aligned}
\max _{\substack{t>0 \\
w \in Z}} \varphi\left(t u_{\varepsilon}+w\right) & \leq \frac{1}{N}\left(\frac{\int_{\Omega}\left|\nabla u_{\varepsilon}\right|^{2}-\lambda\left|u_{\varepsilon}\right|^{2} d x}{\left(\int_{\Omega}\left|u_{\varepsilon}\right|^{2^{*}}|x|^{\alpha} d x\right)^{2 / 2^{*}}}\right)^{N / 2}+O\left(\varepsilon^{N \frac{N-2}{N+2}}\right) \\
& <\frac{1}{N} S^{\frac{N}{2}} .
\end{aligned}
$$

Proof of Theorem 1.1 Propositions 3.1 and 3.2 imply the existence of $u \in \mathcal{N}$ such that $\varphi(u)=c$. In particular, $D \varphi(u)=0$ on the tangent space $T_{u} \mathcal{N}$. Since we have shown that $\mathcal{N}$ is a natural constraint, $u$ is a free critical point of $\varphi$.

Remark 3.2. When $\lambda>\lambda_{1}$, it is very easy to show that our solutions must change sign. Actually, just test (1.1) against $e_{1}$, and conclude that $u$ cannot have the same sign everywhere.

In dimension $N=4$, we can prove the following variant of Theorem 1.1 .

Theorem 3.1. Assume $N=4$ and that $\lambda$ is not a Dirichlet eigenvalue of the Laplace operator. Then, for every $\alpha>0$ sufficiently small, there exists (at least) a ground-state solution to problem (1.1).

The proof is achieved by an easy modification of the previous arguments. It suffices to take into accounts the different asymptotic behavior of the instanton in dimension four.

\section{Additional properties of ground-state solutions}

As in 21], we can prove that ground-state solutions of (1.1) have more properties than being just solutions.

Proposition 4.1. Assume again that $\lambda_{m} \leq \lambda<\lambda_{m+1}$. Then any point $u \in \mathcal{N}$ such that $\varphi(u)=c$ is a critical point of $\varphi$ with Morse index $m+1$.

Proof. $\mathcal{N}$ is a smooth manifold of codimension $m+1$. With the notation introduced with Proposition 2.1, we can write $T_{u} \mathcal{N}=(D F(u))^{-1}(0)$. Since $u$ minimizes $\varphi$ on $\mathcal{N}$, the hessian of $\varphi$ at $u$ is positive definite on $T_{u} \mathcal{N}$. We conclude that the Morse index of $u$ is at most $m+1$. But the proof of Proposition 2.1] shows that this Morse index is at least $m+1$, and the proof is complete. Since $\Omega$ is a radial domain, we might wonder if its symmetry is inherited by ground-state solutions. We do not have a complete answer, as in the situation $\alpha=0$ treated by [21]. However, we can still prove that ground-state solutions are foliated Schwarz symmetric functions. We recall the precise definition for the reader's sake. 
Definition 4.1. A function $u$ on a radial domain is foliated Schwarz symmetric if there exists a unit vector $p \in \mathbb{R}^{N}$ such that $u$ is a function of the distance from the origin and of the angle with the straight line along $p$ only. In other words, $u(x)$ depends only on $|x|$ and on $\arccos \frac{x \cdot p}{|x|}$.

When $\lambda_{1} \leq \lambda<\lambda_{2}$, the constraint $\mathcal{N}$ is actually radially symmetric, by standard results about the symmetry of the first eigenfunction $e_{1}$. As the next results shows, in this situation we can gain more symmetry also for ground-state solutions.

Proposition 4.2. Let $m=1$, i.e. $\lambda_{1} \leq \lambda<\lambda_{2}$. If $u \in \mathcal{N}$ satisfies $\varphi(u)=\inf _{\mathcal{N}} \varphi=$ $c$, then $u$ is foliated Schwarz symmetric.

Proof. Under our assumptions, we remark that

$$
\begin{aligned}
\mathcal{N} & =\left\{u \in H \backslash\{0\} \mid \begin{array}{l}
\int_{\Omega}|\nabla u|^{2}-\lambda u^{2}-|x|^{\alpha} u^{2^{*}}=0 \\
\int_{\Omega} \nabla u \cdot \nabla e_{1}-\lambda u e_{1}-|u|^{2^{*}-2} u e_{1}=0
\end{array}\right\} \\
& =\left\{u \in H \backslash\{0\} \mid \begin{array}{ll}
\int_{\Omega}|\nabla u|^{2}-\lambda u^{2}-|x|^{\alpha} u^{2^{*}}=0 \\
\int_{\Omega}\left(\lambda_{1}-\lambda\right) u e_{1}-|u|^{2^{*}-2} u e_{1}=0
\end{array}\right\}
\end{aligned}
$$

We recall that $e_{1}$, the first eigenfunction of the Laplace operator on $\Omega$, is radially symmetric and positive. Let $u$ be as in the statement, and pick $x_{0} \in \Omega \backslash\{0\}$ with

$$
u\left(x_{0}\right)=\max \left\{u(x)|x \in \bar{\Omega},| x|=| x_{0} \mid\right\} .
$$

For $p=x_{0} /\left|x_{0}\right|$, we define $\mathcal{H}_{p}$ as the set of all closed halfspaces $K$ such that $0 \in \partial K$ and $p$ lies in the interior of $K$. For each $K \in \mathcal{H}_{p}$, there is a reflection map $\sigma_{K}$ across $K$. We need to prove (see [ㅁ, Lemma 4.2]) that

$$
u(x) \geq u\left(\sigma_{K}(x)\right), \quad \text { for all } x \in K \cap \Omega .
$$

So, fix any $K \in \mathcal{H}_{p}$ and consider the polarization of $u$ with respect to $K$, defined by the formula

$$
u_{K}(x)= \begin{cases}\max \left\{u(x), u\left(\sigma_{K}(x)\right)\right\}, & \text { if } x \in \Omega \cap K \\ \min \left\{u(x), u\left(\sigma_{K}(x)\right)\right\}, & \text { if } x \in \Omega \backslash K .\end{cases}
$$

It is known that

$$
\int_{\Omega}\left|\nabla u_{K}\right|^{2}=\int_{\Omega}|\nabla u|^{2}, \quad \int_{\Omega}\left|u_{K}\right|^{q}=\int_{\Omega}|u|^{q}
$$

for every $1 \leq q \leq+\infty$. Since $e_{1}$ is radially symmetric, we also have

$$
\int_{\Omega} u_{K} e_{1}=\int_{\Omega} u e_{1}, \quad \int_{\Omega}|x|^{\alpha}\left|u_{H}\right|^{q-2} u_{K} e_{1}=\int_{\Omega}|x|^{\alpha}|u|^{q-2} u e_{1},
$$

for every $2 \leq q \leq+\infty$. We refer the interested reader to [4, Section 2]. As a consequence of these invariance properties, $u_{K} \in \mathcal{N}$ and $\varphi\left(u_{K}\right)=\varphi(u)=c$. Standard 
methods of elliptic regularity theory implies that both $u$ and $u_{K}$ are classical solutions of (1.1). Set $w=u_{K}-u$, and notice that $w \geq 0$ in $\Omega \cap K$; moreover, $w$ solves the Dirichlet problem

$$
\begin{cases}-\Delta w=q(x) w & \text { in }(K \cap \Omega)^{\circ} \\ w=0 & \text { on } \partial(K \cap \Omega),\end{cases}
$$

where $(K \cap \Omega)^{\circ}$ stands for the interior of $K \cap \Omega$. Here,

$$
q(x)=\lambda+\left(2^{*}-1\right) \int_{0}^{1}|x|^{\alpha}\left|(1-s) u(x)+s u_{K}(x)\right|^{2^{*}-2} d s
$$

for every $x \in \Omega \cap K$. But $q \in L^{\infty}(\Omega \cap K)$, and the strong maximum principle tells us that either $w>0$ in $(\Omega \cap K)^{\circ}$ or $w$ identically vanishes. But $x_{0} \in(\Omega \cap K)^{\circ}$ and $w\left(x_{0}\right)=u_{K}\left(x_{0}\right)-u\left(x_{0}\right)=0$, and thus $w=0$ everywhere. Hence $u_{K}=u$, and (2.1) is proved.

Remark 4.1. We observe that the previous proof is independent of the size of $\alpha$. Unlike [21], we are not able to exclude that $u$ is radially symmetric. Our equation contains the increasing weight $|\cdot|^{\alpha}$, and, as far as we know, there is no precise estimate for the Morse index of radially symmetric solutions of (1.1). See also [22, Section 6.2] for a recent survey on symmetry of solutions for similar equations.

\section{Final comments}

Roughly speaking, the Dirichlet problem

$$
\begin{cases}-\Delta u=\lambda u+|u|^{2^{*}-2} u & \text { in } \Omega \\ u=0 & \text { on } \partial \Omega\end{cases}
$$

is the limiting problem for (1.1) as $\alpha \rightarrow 0$. We have proved that many properties of this limiting problem pass on to (1.1) for small values of $\alpha$. Although ours are pertubative results, it seems rather complicated to apply those methods developed in [3], since non-degeneracy of solutions to (1.1) is unknown.

On the other hand, when $\lambda=0$, many authors studied the asymptotic properties of (1.1) as $\alpha \rightarrow+\infty$ : we refer to [19] for seminal results. In our framework, we face a serious obstacle in (3.11). Indeed, one might try to push the spike $x_{\ell}$ of the instanton towards $\partial \Omega$, with a speed possibly related to $\alpha$ as well. However, the denominator $(1-2 \ell)^{2 \alpha / 2^{*}}$ behaves as an exponential function, whilst the numerator is a polynomial perturbation of the best Sobolev constant. We are therefore unable to treat this situation.

Let us try to explain this obstruction. By analogy with Theorem 3.4 of [17], we may believe that the actual limiting problem as $\alpha \rightarrow+\infty$ is

$$
\begin{cases}-\Delta V=\mathrm{e}^{x_{1}}|V|^{2^{*}-2} V & \text { in } \mathbb{R}_{-}^{N} \\ V=0 & \text { on }\left\{x_{1}=0\right\}\end{cases}
$$


where $\mathbb{R}_{-}^{N}=\left\{x \in \mathbb{R}^{N} \mid x_{1}<1\right\}$. Hence, the optimal level for compactness might be larger than $\frac{1}{N} S^{N / 2}$. There would be room for existence of ground state solutions above $\frac{1}{N} S^{N / 2}$, but the instanton cannot suffice. Anyway, we do not have rigorous proofs of these ideas, yet.

\section{References}

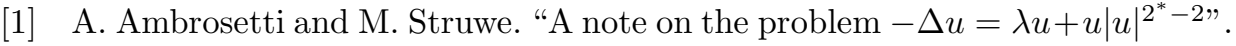
In: Manuscripta Math. 54.4 (1986), pp. 373-379. ISSN: 0025-2611. DOI: 10.1007/BF01168482. URL: http://dx.doi.org/10.1007/BF01168482 (cit. on p. 1).

[2] Antonio Ambrosetti and David Arcoya. An introduction to nonlinear functional analysis and elliptic problems. Progress in Nonlinear Differential Equations and their Applications, 82. Boston, MA: Birkhäuser Boston Inc., 2011, pp. xii+199. ISBN: 978-0-8176-8113-5 (cit. on p. 2).

[3] Antonio Ambrosetti and Andrea Malchiodi. Perturbation methods and semilinear elliptic problems on $\mathbf{R}^{n}$. Vol. 240. Progress in Mathematics. Basel: Birkhäuser Verlag, 2006, pp. xii+183. ISBN: 978-3-7643-7321-4; 3-7643-7321-0 (cit. on p. 12).

[4] Thomas Bartsch, Tobias Weth, and Michel Willem. "Partial symmetry of least energy nodal solutions to some variational problems". In: J. Anal. Math. 96 (2005), pp. 1-18. ISSN: 0021-7670. DOI:10.1007/BF02787822, URL: http://dx.doi.org/10.1007/BF02787822 (cit. on p. 11).

[5] Haïm Brézis and Louis Nirenberg. "Positive solutions of nonlinear elliptic equations involving critical Sobolev exponents". In: Comm. Pure Appl. Math. 36.4 (1983), pp. 437-477. ISSN: 0010-3640. DOI: 10.1002/cpa.3160360405. URL: http://dx.doi.org/10.1002/cpa.3160360405 (cit. on pp. 1, 6] 7).

[6] Friedemann Brock. "Symmetry and monotonicity of solutions to some variational problems in cylinders and annuli". In: Electron. J. Differential Equations (2003), No. 108, 20 pp. (electronic). ISSN: 1072-6691 (cit. on p. 11).

[7] Daomin Cao and Shuangjie Peng. "The asymptotic behaviour of the ground state solutions for Hénon equation". In: J. Math. Anal. Appl. 278.1 (2003), pp. 1-17. ISSN: 0022-247X. DOI:10.1016/S0022-247X (02) 00292-5. URL: http://dx.doi.org/10.1016/S0022 (cit. on p. 2).

[8] Daomin Cao, Shuangjie Peng, and Shusen Yan. "Asymptotic behaviour of ground state solutions for the Hénon equation". In: IMA J. Appl. Math. 74.3 (2009), pp. 468-480. ISSN: 0272-4960. DOI: 10.1093/imamat/hxn035, URL: http://dx.doi.org/10.1093/imamat/hxn035 (cit. on p. 2).

[9] A. Capozzi, D. Fortunato, and G. Palmieri. "An existence result for nonlinear elliptic problems involving critical Sobolev exponent". In: Ann. Inst. H. Poincaré Anal. Non Linéaire 2.6 (1985), pp. 463-470. ISSN: 0294-1449. URL: http://www.numdam.org/item?id=AIHPC_1985__2_6_463_0 (cit. on p. 1). 
[10] Mónica Clapp and Tobias Weth. "Multiple solutions for the Brezis-Nirenberg problem". In: Adv. Differential Equations 10.4 (2005), pp. 463-480. ISSN: 10799389 (cit. on p. 11).

[11] Filippo Gazzola and Bernhard Ruf. "Lower-order perturbations of critical growth nonlinearities in semilinear elliptic equations". In: Adv. Differential Equations 2.4 (1997), pp. 555-572. IsSN: 1079-9389 (cit. on p. 11).

[12] Norimichi Hirano. "Existence of positive solutions for the Hénon equation involving critical Sobolev terms". In: J. Differential Equations 247.5 (2009), pp. 1311-1333. ISSN: 0022-0396. DOI:10.1016/j.jde.2009.06.008 URL:http://dx.doi.org/10. (cit. on p. 2).

[13] Wei Long and Jianfu Yang. "Existence for critical Hénon type equations". In: Advances in Differential Equations (2011) (cit. on pp. 2, 6).

[14] Benedetta Noris and Gianmaria Verzini. "A remark on natural constraints in variational methods". 2011 (cit. on p. 3).

[15] A. Pankov. "Periodic Nonlinear Schrödinger equation with application to photonic crystals". In: Milan J. Math. 73 (2005), pp. 259-287 (cit. on pp. 2] 3).

[16] Enrico Serra. "Non radial positive solutions for the Hénon equation with critical growth". In: Calc. Var. Partial Differential Equations 23.3 (2005), pp. 301326. ISSN: 0944-2669. DOI: 10.1007/s00526-004-0302-9. URL:http://dx.doi.org/10.1007/s00 (cit. on p. 6).

[17] Didier Smets and Michel Willem. "Partial symmetry and asymptotic behavior for some elliptic variational problems". In: Calc. Var. Partial Differential Equations 18.1 (2003), pp. 57-75. ISSN: 0944-2669. DOI: 10.1007/s00526-002-0180-y. URL: http://dx.doi.org/10.1007/s00526-002-0180-y (cit. on p. 12).

[18] Didier Smets, Michel Willem, and Jiabao Su. "Non-radial ground states for the Hénon equation". In: Commun. Contemp. Math. 4.3 (2002), pp. 467-480. Issn: 0219-1997. DOI: $10.1142 /$ S0219199702000725 URL: http://dx.doi.org/10.1142/S02191997020 (cit. on p. 2).

[19] Didier Smets, Michel Willem, and Jiabao Su. "Non-radial ground states for the Hénon equation". In: Commun. Contemp. Math. 4.3 (2002), pp. 467-480. ISSN: 0219-1997. DOI: 10.1142/S0219199702000725, uRL:http://dx.doi.org/10.1142/S02191997020 (cit. on p. 12).

[20] Andrzej Szulkin and Tobias Weth. "Ground state solutions for some indefinite variational problems". In: J. Funct. Anal. 257.12 (2009), pp. 3802-3822. IsSn: 0022-1236. DOI: 10.1016/j.jfa.2009.09.013. URL:http://dx.doi.org/10.1016/j.jfa.2009.0 (cit. on p. 4).

[21] Andrzej Szulkin, Tobias Weth, and Michel Willem. "Ground state solutions for a semilinear problem with critical exponent". In: Differential Integral Equations 22.9-10 (2009), pp. 913-926. ISSN: 0893-4983 (cit. on pp. 2, 3] 5, 6, 10, 12). 
[22] Tobias Weth. "Symmetry of solutions to variational problems for nonlinear elliptic equations via reflection methods". In: Jahresber. Dtsch. Math.-Ver. 112.3 (2010), pp. 119-158. ISSN: 0012-0456 (cit. on p. 12). 